\title{
Controlling the Carrier-Envelope Phase of Raman-Generated Periodic Waveforms
}

\author{
Zhi-Ming Hsieh, ${ }^{1,2}$ Chien-Jen Lai, ${ }^{2, *}$ Han-Sung Chan, ${ }^{3}$ Sih-Ying Wu, ${ }^{2}$ Chao-Kuei Lee, ${ }^{4}$ Wei-Jan Chen, ${ }^{2}$ Ci-Ling Pan, ${ }^{3,5}$ \\ Fu-Goul Yee, ${ }^{1}$ and A. H. Kung ${ }^{2,3, \dagger}$ \\ ${ }^{1}$ Department of Physics, National Taiwan University, Taipei, Taiwan \\ ${ }^{2}$ Institute of Atomic and Molecular Sciences, Academia Sinica, Taipei, Taiwan \\ ${ }^{3}$ Department of Photonics and Institute of Electro-Optical Engineering, National Chiao Tung University, Hsinchu, Taiwan \\ ${ }^{4}$ Department of Photonics, National Sun-Yat-Sen University, Kaohsiung, Taiwan \\ ${ }^{5}$ Department of Physics and Institute of Photonics Technologies, National Tsing Hua University, Hsinchu, Taiwan
}

(Received 14 March 2009; published 27 May 2009)

\begin{abstract}
We demonstrate control of the carrier-envelope phase of ultrashort periodic waveforms that are synthesized from a Raman-generated optical frequency comb. We generated the comb by adiabatically driving a molecular vibrational coherence with a beam at a fundamental frequency plus its second harmonic. Heterodyne measurements show that full interpulse phase locking of the comb components is realized. The results set the stage for the synthesis of periodic arbitrary waveforms in the femtosecond and subfemtosecond regimes with full control.
\end{abstract}

DOI: 10.1103/PhysRevLett.102.213902

Ultrashort subfemtosecond pulses with attosecond timing are essential for probing the evolution of electronic processes in atoms and molecules. The ultimate goal of these studies is to achieve coherent control of electronic motion in matter with light. To reach this goal, it requires developing the ability to generate light pulses with an octave-spanning bandwidth and having the ability to shape the pulses. This means that the phase and amplitude of every component of the pulse spectrum must be adjustable and controllable [1]. Recently, several groups have demonstrated that a convenient way to generate a multioctave optical spectrum is to use molecular modulation whereby two narrow band lasers slightly detuned from a Raman transition are used to drive a molecular motion adiabatically [2-5]. The molecular modulation process provides a broad comb of equally spaced frequencies. When these frequencies are phased properly, a Fourier-synthesized periodic train of single-cycle and subcycle pulses is produced [6,7]. In addition, when the frequencies $\omega_{q}$ of the driving lasers satisfy the commensurate condition that $\omega_{q}$ is an integral multiple of $\omega_{m}$, where $\omega_{m}$ is the frequency difference of the two driving lasers, the carrier-envelope phase (CEP) of each pulse in the train will remain constant throughout the duration of the pulse train $[7,8]$. Yet, in all of the experiments reported to date, the CEP of the Raman-generated pulse trains has varied freely from one set of nanosecond driving pulses to the next. This is because the two driving pulses used are of vastly different frequencies. They have a random phase difference between them. Reliable waveforms cannot be synthesized consistently unless the CEP can be controlled [9]. The dire consequence of lacking this control is that any ultrafast application of the pulse train will require simultaneous measurement of its CEP, compounding the complexity of already difficult experiments to render such applications very unattractive.
PACS numbers: 42.62.Eh, 42.50.Md, 42.65.Ky, 42.65.Re

An obvious solution to this problem is to phase-lock the two driving lasers used in molecular modulation. Yet, while phase locking of two cw lasers is now common practice [10] and the locking of two femtosecond lasers has been demonstrated [11], it remains a tremendous challenge to phase-lock two nanosecond lasers that have a large difference in frequency. Another solution is to derive both driving pulses from one laser source. With this approach, the driving pulses will be mutually coherent at all times. In this Letter, we describe the use of a coherent source whose frequency is slightly detuned from the Raman transition and its second harmonic to drive the $\mathrm{Q}(1)$ Raman coherence in $\mathrm{H}_{2}$. Our measurements reveal that the anti-Stokes orders thus generated are all locked to the relative phase between the fundamental frequency and its second harmonic, leading to a stable static phase for every component of the generated comb at all times. The Fourier synthesis of these frequencies would then result in a periodic train of transform-limited ultrashort pulses whose CEP can be set and controlled at will.

The CEP of frequency combs generated from cw modelocked Ti:sapphire lasers has been controlled both actively $[12,13]$ and passively [14] with high precision. Here we show the control of the CEP of Raman combs generated with nanosecond pulses. Since it is relatively easy to produce multioctave-spanning combs with the Raman approach, this work could set the stage for Ramansynthesized periodic optical waveforms in the femtosecond to subfemtosecond domain to be used in novel applications such as subfemtosecond pump-probe and control in the optical region [15] and particle acceleration, plasma modulation, and molecular orientation that use phase-locked pulse arrays [16-19].

We shall first explain the working principle behind our approach. When a frequency comb is phased for Fourier synthesis to a periodic waveform, the frequency $\omega_{q}$ and the 
phase $\phi_{q}$ of the $q$ th component of the comb follow the relations

$$
\omega_{q}=\omega_{c e o}+q \omega_{m}, \quad \phi_{q}=\phi_{0}+q \phi_{m},
$$

where $\omega_{\text {ceo }}$ is the carrier-offset frequency, $\phi_{0}$ is a static offset, $\omega_{m}$ is the comb frequency spacing, and $\phi_{m}$ is a linear phase difference between adjacent comb components. The synthesized waveform can then be expressed as

$$
\begin{aligned}
E(t) & =e^{i\left(\omega_{c e o} t+\phi_{0}\right)} \sum_{q} A_{q} e^{i q\left(\omega_{m} t+\phi_{m}\right)} \\
& =e^{i \phi_{c e}} \sum_{q} A_{q} e^{i q\left(\omega_{m} t+\phi_{m}\right)} .
\end{aligned}
$$

The CEP $\phi_{c e}$ of the waveform thus has a time-varying component $\omega_{\text {ceo }} t$ and a static component $\phi_{0}$. When the frequencies are commensurate, $\omega_{\text {ceo }}$ vanishes. But the static phase $\phi_{0}$ must still be identical from one pulse to the next in order for the CEP and consequently the waveform to be the same at all times.

In the case of a Raman-generated comb, $\omega_{m}$ is the Raman modulation frequency. If we let $\omega_{p}$ be the lower of the two driving frequencies and when the frequencies are commensurate, then $\omega_{p}=p \omega_{m}$ [i.e., $q=p$ in (1)]. The second driving frequency necessarily equals $\omega_{p+1}=$ $\omega_{p}+\omega_{m}=(p+1) \omega_{m}$ (see Fig. 1). The corresponding input phases are $\phi_{p}$ and $\phi_{(p+1)}$. When the frequencies are adjusted for Fourier synthesis to a periodic waveform, the phases will be $\phi_{p}=\phi_{0}+p \phi_{m}$ and $\phi_{(p+1)}=\phi_{0}+(p+$ 1) $\phi_{m}$. Solving for $\phi_{0}$ gives $\phi_{0}=(p+1) \phi_{p}-p \phi_{(p+1)}$. Hence the "static" phase is not invariant if the input driving phases $\phi_{p}$ and $\phi_{(p+1)}$ are not locked. On the other hand, by choosing $p=1$, the second frequency is necessarily $2 \omega_{m}$, which can be obtained by phase-matched second-harmonic generation (SHG) from $\omega_{m}$. This results in $\phi_{2}$ equaling $2 \phi_{1}+\pi / 2$ from the nonlinear optical conversion process. The linear phase difference $\phi_{m}$ now is $\phi_{1}+\pi / 2$, and the phases of the Raman-generated comb can be adjusted to the form

$$
\phi_{q}=\phi_{1}+(q-1)\left(\phi_{2}-\phi_{1}\right)=-\pi / 2+q\left(\phi_{1}+\pi / 2\right) .
$$

Comparing (1) and (3), we see that the CEP is now equal to $-\pi / 2$ that is independent of $\phi_{1}$ and is fixed at all times
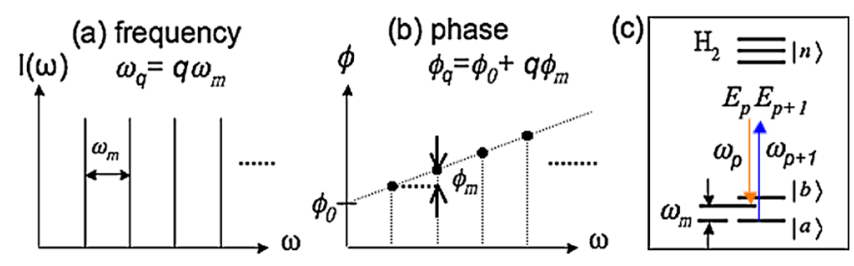

FIG. 1 (color online). Graphic representation of Eq. (1) for the commensurate case to show the relationship between (a) the frequency and (b) the phase among the comb components. (c) relates the frequency terms in (1) to the driving frequencies in the Raman case. barring external distortions. Meanwhile, it will be possible to set and control the CEP by inserting a phase shifter in front of the Raman medium that systematically varies $\phi_{2}-2 \phi_{1}$, the CEP.

We performed a set of experiments to verify this approach of controlling the CEP in a Raman-generated frequency comb. The experimental setup is shown in Fig. 2. A beam at the fundamental frequency $\omega_{1}$ is obtained by difference mixing of the transform-limited outputs of a pulsed dye laser and a pulsed Ti:sapphire laser. Both lasers are operating at $30 \mathrm{~Hz} . \omega_{1}$ is tuned to $4155.235 \mathrm{~cm}^{-1}$ $\left(\lambda_{1}=2406 \mathrm{~nm}\right)$, which is at an offset of $1.0 \mathrm{GHz}$ from the $\mathrm{Q}(1)$ Raman transition of room temperature $\mathrm{H}_{2}$ [7]. A type I phase-matched $\mathrm{LiNbO}_{3}$ crystal then frequency doubles a portion of the energy of $\omega_{1}$ to $2 \omega_{1}$. The remaining $\omega_{1}$ is polarization-rotated to match the polarization of the second harmonic. The fundamental and the secondharmonic beams are focused to $\sim 15 \mathrm{GW} / \mathrm{cm}^{2}$ each at the center of a cell filled with room-temperature $\mathrm{H}_{2}$ at 1200 mbar. Since the driving beams are at the lowest orders of the Raman process, only higher-order anti-Stokes radiation can be generated. But some of the $2 \omega_{1}$ energy will still be channeled to the Stokes radiation at $\omega_{1}$.

The incident fundamental and the second-harmonic pulse energies were 2.3 and $4 \mathrm{~mJ}$, respectively. The pulse durations were both $\sim 3.5 \mathrm{~ns}$ FWHM. At least six new collinearly propagating anti-Stokes wavelengths are generated. Approximately $33 \%$ of the $2 \omega_{1}$ energy is converted to other frequencies: $60 \%$ to the Stokes and $40 \%$ to the anti-Stokes for a net of $13 \%$ energy conversion to new frequencies. This conversion is consistent with measurements made previously using higher driving frequencies [5], and the result indicates that molecular modulation is just as effective when driven with frequencies at the lowest orders.

The Raman comb components generated above are in essence multiple harmonics of the fundamental frequency. They satisfy the relationship $\omega_{i}+\omega_{j}=\omega_{k}$, where $(i+$ $j)=k$ for any $k$. Consequently, any component $\omega_{k}$ of the output can be used as a local oscillator to heterodyne with the nonlinear sum-mixed output of $\omega_{i}$ and $\omega_{j}$ to determine the phase of the comb components relative to each other and for compensation and interactive optimization of the

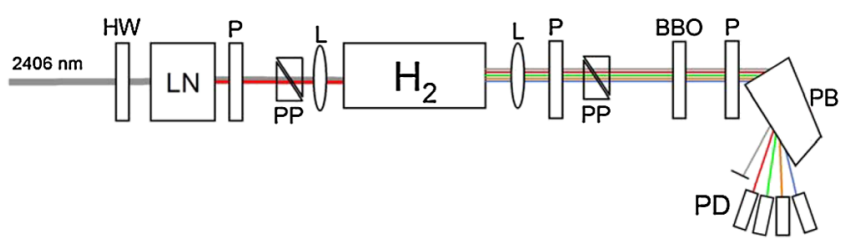

FIG. 2 (color online). Schematic of the experimental setup. $\mathrm{HW}$, half-wave plate; $\mathrm{LN}, \mathrm{LiNbO}_{3}$ crystal; $\mathrm{P}$, polarizer; $\mathrm{PP}, \mathrm{CaF}_{2}$ prism pair; L, lens; $\mathrm{BBO}, \beta$-barium borate; $\mathrm{PB}$, Pellin-Broca prism; $\mathrm{PD}, \mathrm{Si}$ photodiode. Beams from the $\mathrm{H}_{2}$ cell are shown here as different colored beams. They are in fact overlapped in space before the PB prism. 
relative phases of the components [20]. Once compensated, the comb phases follow the relationship given in Eq. (3), permitting the control of the CEP to facilitate waveform synthesis with these frequencies.

Now we demonstrate experimentally the mutual phase coherence among all of the comb components and CEP control by employing the first five frequency components of the generated comb. The comb wavelengths used are nominally at 2406, 1203, 802, 602, and $481 \mathrm{~nm}$, respectively. The relative phases of the 2406 and $1203 \mathrm{~nm}$ beams are altered during the experiment by changing their optical path length via lateral translation of one prism of the first prism pair placed in front of the $\mathrm{H}_{2}$ cell. Output from the $\mathrm{H}_{2}$ cell is collimated and sent through a $3.3 \mathrm{~mm}$ thick $\beta$-barium borate (BBO) crystal placed where the synthesized pulses would later be used. The BBO crystal is cut at nominally $\theta=21.2^{\circ}$ and $\phi=0^{\circ}$ for type I phase-matched SHG or sum frequency mixing (SFG) of the five frequencies. Another prism pair is placed in front of the BBO crystal to compensate the phase shifts caused by external factors other than those due to the first prism pair and the BBO summing process. The Raman frequencies and summed frequencies generated from the BBO crystal are then dispersed by a Pellin-Broca prism and detected by a set of photodiodes. Since the polarization of the summed output is orthogonal to that of the Raman output, a polarizer is used to project the polarization of the two onto a common axis to enable and maximize the heterodyning. The heterodyne signals are sent to a boxcar signal averager and then recorded into a PC for processing. With five frequency components, four measurements are needed for optimization of their relative phases. Meanwhile, there are six independent mixing combinations with the five beams. We elected to use the following four: (a) SHG of 2406 to $1203 \mathrm{~nm}$, (b) SFG of 2406 and 1203 to $802 \mathrm{~nm}$, (c) SFG of 2406 and 802 to $602 \mathrm{~nm}$, and (d) SFG of 2406 and 602 to $481 \mathrm{~nm}$ for phase diagnostics and optimization.

The phase-matching angle is first adjusted to maximize the output of process (a), and the corresponding heterodyne signal is maximized by rotating the third polarizer. The resulting signal is then recorded as the phase difference $\phi_{2}-2 \phi_{1}$ is scanned by translating laterally one prism in the first prism pair using a microstepper. In our setup, a $360 \mu \mathrm{m}$ translation of the prism corresponds to a relative phase difference of $2 \pi \mathrm{rad}$ so that the accuracy of our relative phase determination is easily better than $0.005 \pi$. This procedure is repeated for processes (b)-(d) with each scan starting at the same prism setting. The result of the scans is shown in Figs. 3(a)-3(d). All four heterodyne signals show the same period of oscillation versus the relative $\phi_{2}-2 \phi_{1}$. This agrees with the theoretical expectation from the fact that the Raman local oscillator and the sum-mixed output from the BBO are identical harmonics of the same fundamental frequency. Process (a) involves only $\omega_{1}$ and $2 \omega_{1}$ so that its heterodyne signal need not depend on the Raman conversion. This signal is used to calibrate the relative prism position as a function of the

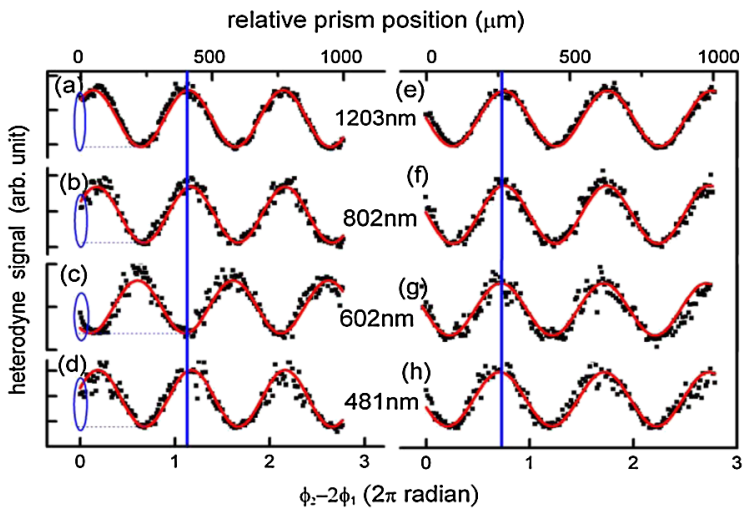

FIG. 3 (color online). Heterodyne signals from sum mixing in a BBO crystal as a function of the translation of a prism in the first prism pair for the processes: (a) SHG of 2406 to $1203 \mathrm{~nm}$, (b) SFG of 2406 and 1203 to $802 \mathrm{~nm}$, (c) SFG of 2406 and 802 to $602 \mathrm{~nm}$, and (d) SFG of 2406 and 602 to $481 \mathrm{~nm}$. All scans of the prism position begin at the same " 0 " prism position. The signal of (a) is used to calibrate the relative prism position shown on the top to the phase difference $\phi_{2}-2 \phi_{1}$ shown on the bottom. Each data point is the average of 30 laser shots. Each trace took about 5 minutes to complete. The squares are data points. The red solid curves are sinusoidal fits to the data. The elongated circles and the blue vertical lines highlight that there is a difference in the initial relative phases in (a)-(d).

phase difference $\phi_{2}-2 \phi_{1}$, which in turn is used to calibrate the horizontal axis of Figs. 3(b)-3(d).

As shown, the magnitude of the heterodyne signal at the starting position of each scan and the location of the scan maxima are different for each process. This indicates that, although the relative phases among the five beams are fixed, they are not matched at the input face of the crystal. The mismatch originates from dispersions and shifts encountered during beam propagation. We compensate for these external factors by adjusting the path length with a second prism pair placed in front of the BBO crystal. The number of adjustments needed is based on the phase difference calculated from the magnitude of the initial heterodyne signals. The signals after phase compensation are shown in Figs. 3(e)-3(h). They show that the phases are aligned (at the BBO input face). All five components are now phased. Their phases are described by (3). The heterodyne signal reaches a maximum or minimum whenever $\phi_{2}-2 \phi_{1}=-(2 n+1) \pi / 2$ for any integer $n$ and the signal inflection points are at $\phi_{2}-2 \phi_{1}=n \pi$. Now the superposition of the frequencies forms a periodic train of single-cycle pulses at the input face of the BBO crystal.

We see from (1) and (3) that the CEP of each synthesized waveform is equal to $\phi_{2}-2 \phi_{1}$. This suggests that the CEP can be set and controlled solely by varying the phases $2 \phi_{1}$ and $\phi_{2}$ by changing the position of the moving prism in the first prism pair. This can be verified by monitoring the heterodyne signals. Meanwhile, during the above experiment we found that the phase-matching angles for processes (b) and (c) are unresolvable (within $0.01^{\circ}$ ) for 

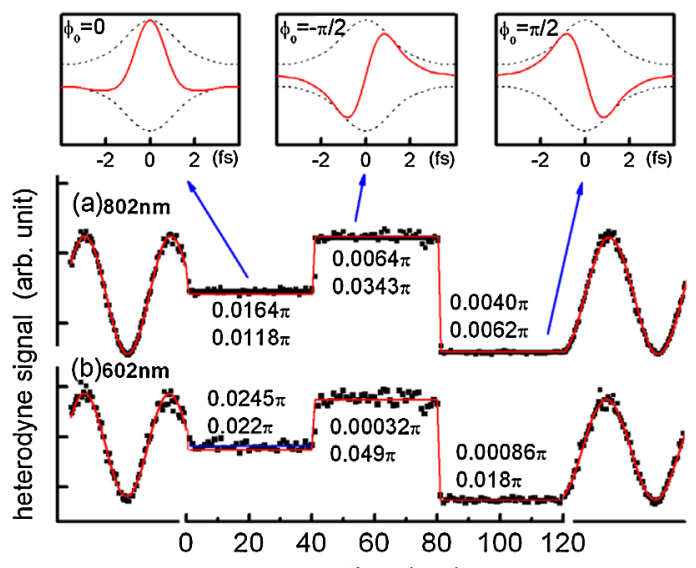

scan time (sec)

FIG. 4 (color online). Heterodyne signals at (a) 802 and (b) $602 \mathrm{~nm}$ used to demonstrate CEP control. The squares are data obtained by laterally translating the prism in the first prism pair. The red solid curves are sinusoidal fits to the data. Red horizontal lines represent the expected signal based on the CEP value to which it is set. The upper set of numbers adjacent to the red lines is the difference of the mean of the measured relative phase and the set value. The lower set of numbers is 1 standard deviation of the relative phase determined from the respective signal fluctuations. The panels on top of the figure show the corresponding electric field waveforms synthesized based on the measured energies of $1.9,1.24,0.3,0.05$, and $0.005 \mathrm{~mJ} /$ pulse, respectively, at 2406, 1203, 802, 602, and $481 \mathrm{~nm}$ and the CEP set for that location. Only the scan time is shown on the horizontal axis.

our BBO crystal so that detection at 602 and $802 \mathrm{~nm}$ can be done simultaneously. We begin the measurement by first scanning the prism in the first prism pair to calibrate the prism position. Then we stop the scan at an arbitrary prism position, move the prism to a position that corresponds to a calculated CEP value of zero based on the calibration, and record the heterodyne signals for $40 \mathrm{sec}$. We then repeat the move-and-record procedure for CEP values of $-\pi / 2$ and $\pi / 2$, respectively. Finally, we scan the prism again to ascertain that the last CEP truly corresponds to the value to which we set it. By executing this sequence, we are demonstrating the setting and controlling of the CEP of the electromagnetic waveform. The results shown in Fig. 4 are the average of 6 scan-stop sequences. The experimental mean values of the CEP agree to within $0.025 \pi \mathrm{rad}$ of the set values. The largest source of error in setting the CEP is in the accuracy in the calibration of the prism position. Also shown in the figure are the synthesized waveforms that correspond to the measured intensities and phases of the comb components used in the measurement.

This experiment demonstrates that, once set, the CEP remains constant for thousands of nanosecond pulses and could be a lot more. The standard deviation of the heterodyne signal provides a measure of the stability of the CEP. Amplitude fluctuations as well as relative phase fluctuations all contribute to the fluctuation in the heterodyne signal. If we conservatively attribute the fluctuations as coming entirely from relative phase fluctuations, we see that the worst-case CEP stability is $0.049 \pi$ rad. In reality, the amplitude fluctuation of the comb components from pulse to pulse is quite large $( \pm 15 \%)$ so that in fact the interpulse long term CEP stability is a lot better than $0.049 \pi$. It is worth noting that this stability was obtained without making special attempts to stabilize the optical mounts or optical table used in the experiment.

In summary, we have demonstrated control of the carrier-envelope phase of ultrashort periodic waveforms that were synthesized from a Raman optical frequency comb generated by using the fundamental frequency and its second harmonic to drive a molecular coherence. Since molecular modulation is a demonstrated approach that produces multioctave frequency combs, this work opens the way for using Raman-generated ultrashort pulse trains to probe phase-dependent dynamics and makes a major step forward toward realizing the synthesis of subfemtosecond periodic optical waveforms of arbitrary shape.

The authors thank S. E. Harris and S. Goda for insightful discussions. This work was supported by the Thematic Projects Program of the Academia Sinica and by the National Science Council of Taiwan.

*Present address: EECS Department, MIT, MA, USA.

${ }^{\dagger}$ Corresponding author. akung@pub.iams.sinica.edu.tw

[1] C.-B. Huang et al., Laser Photon. Rev. 2, 227 (2008).

[2] S. E. Harris and A. V. Sokolov, Phys. Rev. Lett. 81, 2894 (1998).

[3] A. V. Sokolov et al., Phys. Rev. Lett. 85, 562 (2000).

[4] J. Q. Liang et al., Phys. Rev. Lett. 85, 2474 (2000).

[5] S. W. Huang, W.-J. Chen, and A. H. Kung, Phys. Rev. A 74, 063825 (2006).

[6] M. Y. Shverdin et al., Phys. Rev. Lett. 94, 033904 (2005).

[7] W.-J. Chen et al., Phys. Rev. Lett. 100, 163906 (2008).

[8] T. Suzuki, M. Hirai, and M. Katsuragawa, Phys. Rev. Lett. 101, 243602 (2008).

[9] S. N. Goda et al., Opt. Lett. 30, 1222 (2005).

[10] J. Ye and J. L. Hall, Opt. Lett. 24, 1838 (1999).

[11] R. K. Shelton et al., Science 293, 1286 (2001).

[12] D. J. Jones et al., Science 288, 635 (2000).

[13] A. Apolonski et al., Phys. Rev. Lett. 85, 740 (2000).

[14] A. Baltuška, T. Fuji, and T. Kobayashi, Phys. Rev. Lett. 88, 133901 (2002).

[15] T. Nakajima and S. Watanabe, Phys. Rev. Lett. 96, 213001 (2006).

[16] T. Plettner et al., Phys. Rev. Lett. 95, 134801 (2005).

[17] X. Zhang et al., Nature Phys. 3, 270 (2007).

[18] T. V. Truong and Y. R. Shen, Phys. Rev. Lett. 99, 187802 (2007).

[19] S. Adachi and T. Kobayashi, Phys. Rev. Lett. 94, 153903 (2005).

[20] T. W. Hansch, Opt. Commun. 80, 71 (1990). 